\section{To win friends and influence people}

\section{Ian Stewart}

The Elements of Graphing Data. By William S. Cleveland. Wadsworth:1985. Pp.323. Hbk \$27.95, £37.80; pbk \$18.95, $£ 25.60$.

"IN 1980," says the author, "I began a study of graphs in scientific publications ... In one study, I read the articles and reports of the 1980 Volume 207 of Science . . 3 $30 \%$ of the graphs in the volume had at least one of four types of specific problems." Those problems were: something on the graph but not explained, parts of the graph being visually indistinguishable, and mistakes such as mislabelling or poor reproduction. Deeper problems included poor choice of the form in which to present the data, and indeed poor choice of data. In an effort to combat these problems, and to suggest effective countermeasures, this book discusses the principles and methods of graphical construction, amply illustrated by examples selected from the literature (with references, which may lose the author a few friends but makes the arguments more cogent).

Scientists spend a large part of their training learning how to do science; and very little learning how to communicate it. Perhaps this is unavoidable, especially given the pressures of crowded curricula; possibly it would make little difference if students attended the odd course on good presentation and effective data display. But, at the very least, they could be encouraged to read a book such as this. Once sensitized to the pitfalls, they might be more prepared to put in the effort required to select a clear and effective means of presenting their results.

Consider even the most standard type of graph, with two axes, some tick marks to show the scale, dots for the data, and lines connecting the dots. Do the data stand out. or are they obscured by the connecting lines? Do the data points overlap and obscure each other? Do the axes fall across data points? Are there too many tick marks? Do the labels clutter the picture and make it hard to grasp the significant features of the data? All these matters require proper attention. Other types of graphical medium. such as the scatterplot, pose other questions.

One especially fraught area is the display of multidimensional data. To graph the relations between ten or twelve different variables can stretch even the most active imagination. Most attempts fail in one way or another. The author suggests the use of colour to improve readability; this is fine in principle, but most journals refuse to include colour because of its cost. The scatterplot matrix - an array of coordinated plots of all possible pairs of variables, one against the other - manages to convey a great deal of information succinctly and clearly.

The danger with principles is that they can be taken too literally. A few horrific examples show what happens when the Darrell Huff Dictum, "always include zero in the scale", is overdone. To cramp the data into a tiny corner of an otherwise blank graph helps nobody. Huff was thinking more about advertising or political propaganda, and when preparing a graph for scientific publication, it should be permissible to assume that the reader has the intelligence to read the numbers on the axes.

If you're not willing to present your data effectively, then presumably you don't really care what they are. If you don't write papers that others can read, why write papers at all? The author's basic message is a simple one: remember that the important thing is the data. Granted this, all else will follow.

Ian Stewart is a Reader in Mathematics at the University of Warwick, Coventry, CV4 $7 A L$, UK.

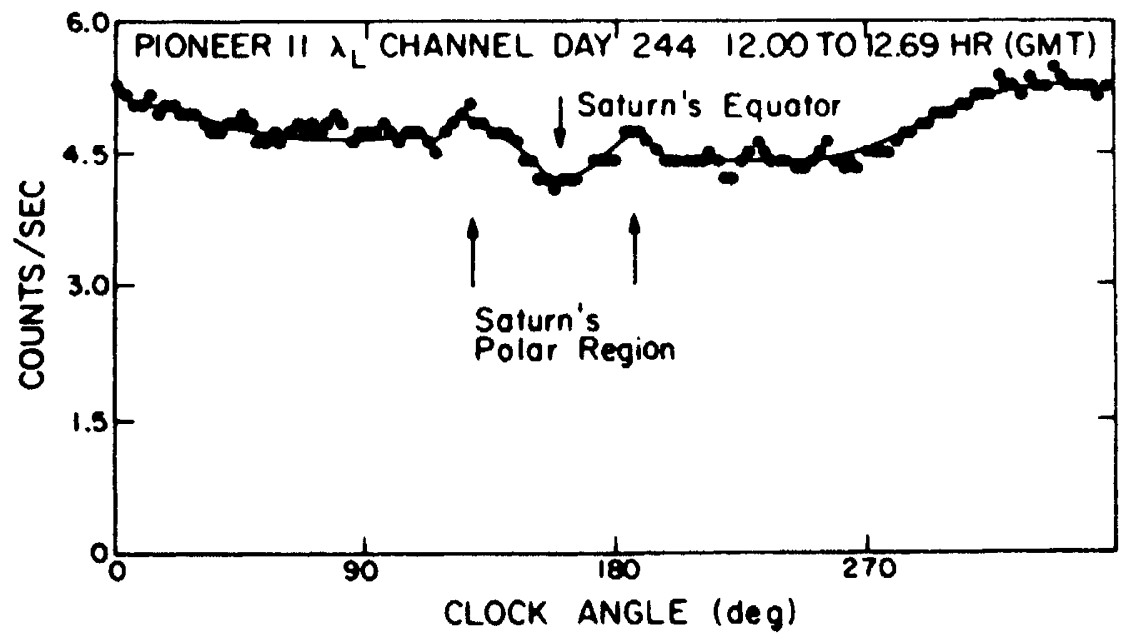

The resolution of this graph is degraded by the inclusion of zero on the vertical scale.

\section{A look around the kitchens}

\section{Alastair Hay}

Controlling Chemicals: The Politics of Regulation in Europe and the United States. By Ronald Brickman, Sheila Jasanoff and Thomas Ilgen. Cornell University Press:1985. Pp.344. \$38.45.

EARLY attempts by some United States Government Agencies to control the availability and use of chemicals were derided by critics, and the chemical industry in particular, as "cook-book" approaches to the subject. Fifteen years later things have changed, but analogy with cooking remains an appropriate way to describe the thesis of this book.

The authors have set out to compare how chemicals are regulated in Britain, West Germany, France and the United States. It is as if four cooks were asked to bake a loaf of bread - starting with the same ingredients, they follow different recipes but end up with a loaf nevertheless. The European cooks keep their techniques to themselves, arguing that their success lies in some secret mix. The American, on the other hand, has an audience to scrutinize all the ingredients, check the recipe and follow each step from mixing to baking.

The same applies to chemical regulation. Europeans prefer a consensus approach, negotiating with interested parties behind closed doors and presenting final versions of regulations which are rarely altered by Parliament. In the United States, however, the procedure is adversarial, with the government, Congress, the courts, public interest groups and industry all having a say in the matter.

While recognizing that the European pattern of involvement is more restrictive, the contention of Brickman and his colleagues is that, despite this lengthy and expensive process, legislation in Europe is as - or more - effective as that in the United States. (To prove the point, a comparative table of regulated substances is appended to the book, showing limits for the four countries.) Their conclusion is drawn from a thorough analysis of the European systems, some features of which, they argue, could well bear consideration by both regulators and legislators in the United States.

There are fundamental differences between the regulatory processes applied in Europe and the United States. Where the scientific evidence on the hazards - and the carcinogenic risk in particular - of a chemical is unclear, the Europeans tend to adopt a policy of 'innocent until proven guilty'. In the United States, however, guilt is assumed, until proven otherwise, and decisions about regulations are made from that standpoint. 